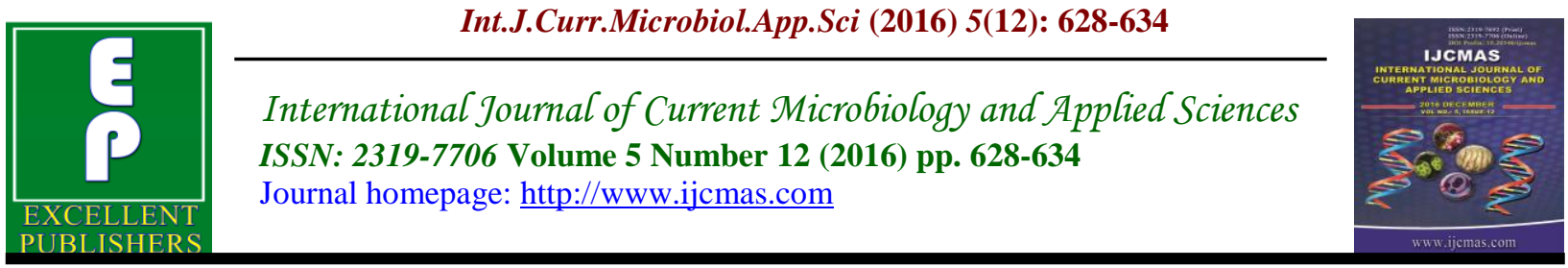

Original Research Article

http://dx.doi.org/10.20546/ijcmas.2016.512.069

\title{
Species Distribution, Biofilm Formation and Antifungal Susceptibility of Candida Isolates in Blood Samples of NICU Patients at Tertiary Care Centre, Amritsar, India
}

\author{
Rajesh Bansal*, Loveena Oberoi, Kanwardeep Singh and Pushpa Devi \\ Department of Microbiology, Government Medical College, Amritsar, India \\ *Corresponding author
}

Keywords

NICU (neonatal intensive care unit); Antifungal susceptibility testing; biofilm.

\section{Article Info}

Accepted:

20 November 2016 Available Online: 10 December 2016

\section{A B S T R A C T}

To study the distribution, biofilm formation and antifungal susceptibility pattern of Candida species isolated from neonatal candidemia patients. Candida infections are a serious problem in neonatal intensive care units leads to increasing morbidity and mortality. A prospective observational study was conducted from September 2014 to June 2016. A total of 313 blood samples received in Microbiology department from NICU patients with suspected Candida infection were collected. Samples were processed by Gram staining, $\mathrm{KOH}$ mount \& culture on SDA and BHI agar. Isolated yeasts were identified and speciated by germ tube test, chlamydospores formation on corn meal agar, color production on CHROM agar, sugar fermentation test and sugar assimilation test. Antifungal susceptibility testing of isolates was performed as per CLSI guidelines. Biofilm production was tested by Tube method and Tissue culture plate method. The data was statistically analysed using the statistical package for Social science (SPSS)/21.0. A total of 60 (19.17\%) samples out 313 blood samples were positive for candida infections. Most common fungal isolate was Candida tropicalis (45\%) followed by Candida albicans (25\%), C. parapsilosis (11.7\%), C. glabrata (11.7\%) and C. krusei (6.7\%.). Maximum resistance was observed to Fluconazole \& Ketoconazole. Antifungal resistant was found to be more in biofilm producers. This Study highlights the emergence of Non albicans Candida as major isolates and increasing antifungal resistance.

\section{Introduction}

Candidiasis in neonatal intensive care units has increased steadily in incidence over the last two decades (Kossoff et al., 1998). The reported incidence ranges between $1.6 \%$ to $9 \%$ in very low birth weight (VLBW) and $10 \%$ to $16 \%$ in extremely low birth weight (ELBW) infants with a clear association with decreasing gestational age (Johnsson et al., 2004). Candida species accounts for $9 \%$ to $13 \%$ of all hospital acquired blood stream infections (BSIs) (Beck-Sague et al., 1994). The incidence and associated mortality due to candidemia can be influenced by several factors including characteristic of the population at risk, standard of the health care facilities available, distribution of Candida species, and prevalence of antifungal resistance. Although $C$. albicans 
has historically been the most frequently isolated species, infections caused by the Non albicans Candida have been diagnosed with increasing frequency in recent years, notably $C$. tropicalis, $C$. glabrata, $C$. parapsilosis. and C. krusei. There is growing evidence suggesting a role of increasing use of azole agents in this epidemiological shift. Several of these NAC species exhibit intrinsic resistance to traditional triazoles like Fluconazole (FLK) and may also demonstrate cross resistance to newer triazoles. Biofilm is an important virulence factor that can lead to antifungal resistance.

\section{Materials and Methods}

A prospective observational study was conducted from September 2014 to June 2016. A total of 313 blood samples received in Microbiology department from NICU patients with suspected Candida infection were collected and processed. 1-2 $\mathrm{ml}$ of blood sample was inoculated directly in a culture system like brain heart infusion agar and later sub cultured onto Sabourauds Dextrose Agar medium. Isolated yeasts were identified \& speciated by germ tube test, chlamydospores formation on corn meal agar, color production on CHROM agar, sugar fermentation test and sugar assimilation test (Chander, 2009).

Antifungal susceptibility testing of the yeast isolates was performed by 'Disc Diffusion Method' including Amphotericin B (100 IU), Fluconazole $(25 \mu \mathrm{g})$, Nystatin $(50 \mu \mathrm{g})$, Ketoconazole $(50 \mu \mathrm{g})$, and Itraconazole $(10 \mu \mathrm{g})$ as per CLSI guidelines (Wayne, 2009).

Reference strains from quality control methods used were,

- Candida parapsilosis ATCC 22019
- Candida albicans ATCC 90028

- Candida tropicalis ATCC 750

- Candida krusei ATCC 6258

Biofilm formation ability of yeast isolates were tested by Tube Adherence Test and Tissue Culture Plate Method.

The data was statistically analysed using the statistical package for Social science (SPSS)/ 21.0 (Copyright (C) SPSS Inc.). Frequency of qualitative variables was calculated and correlation was tested by Chisquare test. Statistical significance was accepted at $\mathrm{p}<0.05$ (Park, 2011).

\section{Results and Discussion}

A total of $60(19.17 \%)$ Candida isolates were obtained from 313 neonatal blood culture samples. Most common fungal isolate was Candida tropicalis 27/60 (45\%) followed by Candida albicans 15/ 60 (25\%), C. parapsilosis $7 / 60$ (11.7\%), C. glabrata 7/60 (11.7\%) and C. Krusei 4/60 (6.7\%).

Early neonatal period (0-6 days) 55/60 (91.67\%) was most common age group followed by infantile period (2-12 month) 3/ $60(5 \%)$ and late neonatal period (7-28) 2/60 (3.33\%) found to be associated with candidemia among NICU patients.

Major risk factors determined in our study were Short for gestational age (SGA)/ Prematurity and low birth weight ( $\leq 2500 \mathrm{~g}$;) 23/60 (38.33\%) each followed by very low birth weight $(\leq 1,500 \mathrm{~g} ;)$ 10/60 (16.67\%), congenital heart disease $3 / 60(5 \%)$ and septicemia 1/60 (1.67\%) Candidemia was found more in male babies 44/60 (73.33\%) than female babies 16/60 (26.67\%).

Fluconazole and Ketoconazole were main drugs found to be resistant among different Candida species. Resistance was more seen 
among Non albicans candida significantly. Maximum resistance towards antifungal drug was seen in Candida krusei and Candida glabrata. Tissue culture plate method 40/60 (66.67\%) was more sensitive than tube method $24 / 60(40 \%)$.

A total of $40(66 \%)$ out of 60 Candida isolates obtained produced biofilm. Only $26.67 \%$ ( 4 of 15) of C. albicans isolates produced biofilm, which was significantly lower than the percentage of all Non albicans Candida species isolates producing. slime (88.89\%, 40 of 45$)$. Strong biofilm production was seen in $C$. krusei and $C$. tropicalis. Weak biofilm production was seen in $C$. albicans. Biofilm producers were more resistant to antifungal drugs.

Candida species are frequently encountered in the normal microbiota of humans, which facilitates them to invade most implanted biomaterials and host surfaces. They are now the fourth leading cause of bloodstream infections in hospitalized patients. In our study, isolation rate of candida isolates from neonatal septicemia cases was $19.67 \%$ (60/313) which was same as several other reports showing frequency of isolation in 13.6- $19.6 \%$ cases. In the present study, Non albicans Candida 45/ 60 (75\%) was predominantly isolated compared to Candida albicans15/60 (25 \%). The predominance of Non albicans Candida species was found to be statistically significant $(\mathrm{p}<0.05)$. This result is collaborated well with the study by Sardana et al., 2012, Xess et al., 2007 and Baradkar et al., 2008. This change in pattern has been partly attributed to increased immune suppression resulting in higher numbers of susceptible immunocompromised patients, hospitalization especially in the NICUs, placement of central venous catheters and prophylactic use of antifungal agents in critically ill patients. Among non albicans candida C. Tropicalis 27/60 (45\%) was major isolate followed by $C$. Parapsilosis 7/60 (11.7\%), C. Glabrata 7/60 (11.7\%) and C. Krusei 4/60 (6.7\%.). This result was found to be statistically significant (p $<0.05)$. C. tropicalis was most commonly isolated in blood as the cause of candidemia in intensive care or neutropenic patients. The striking feature of the present study was the isolation of C. parapsilosis (11.7\%) and C. glabrata (11.7\%) as the second most common among Non-albicans Candida species which was in accordance with study done by Trofa $e t$ al., 2008. While previous study by Narain et al., 2003 and Narang et al., 1998 have documented C. albicans as the most common isolate from neonates. $C$. parapsilosis as an emerging fungal pathogen and a major threat for future especially among neonates in NICU's. Higher affinity of $C$. parapsilosis to adhere on foreign material and ability to form biofilms are important factors for the development of fungemia (Bonassoli et al., 2005).

The most common age group to be infected with candidemia was early neonatal period (0-6 days) ( $91.67 \%)$ which was in accordance to the study done by Juval et al., 2013. In our study this correlation was found to be statistically significant ( $\mathrm{p}<0.05)$. Candidemia was found more in male babies (73.33\%) than female babies (26.67\%).

Short for gestational age (SGA)/prematurity and low birth weight were most common risk factors associated with neonatal candidemia followed by followed by very low birth weight $(\leq 1,500 \mathrm{~g}$;) (16.67\%), congenital heart disease $(5 \%)$ and septicemia (1.67\%). In our study this correlation was found to be statistically significant ( $\mathrm{p}<0.05)$. This was in accordance to studies by Juval et al., 2013 and Narain et al., 2003. Preterm, low birth 
weight babies are more vulnerable to acute fungal sepsis, primarily because of an immature immune system, invasive interventions and prolonged use of antimicrobials that serve as risk factors for fungal colonization. Preceding colonization is an important risk factor for subsequent dissemination and invasive disease.

Antifungal susceptibility testing in our study revealed that Fluconazole ( $80 \%$ ) and Ketoconazole ( $70 \%$ ) were most common antifungal drug found to be resistant which was in accordance to study done by Shin et $a l ., 2002$. Non albicans candida species were more resistant to antifungal drugs. The possibility of increase in the percentage of the resistance to antifungal agents among Non-albicans Species is due to widespread use of antifungal drugs, long-term use of suppressive azoles and the use of short courses of antifungal drugs ( $\mathrm{Ng}$ et al., 2001). Maximum resistance towards various antifungal drugs was found in C. krusei and C. glabrata. Similar results were obtained in study done by Sobel and Babin et al., 2013. In C. krusei, there is intrinsically resistance to azoles.

Table.1 Correlation of antifungal resistance with different Candida species

\begin{tabular}{|l|l|l|l|l|l|l|l|l|l|l|}
\hline \multirow{2}{*}{ Candida species } & \multicolumn{2}{l}{ Ketoconazole } & \multicolumn{2}{l|}{ Fluconazole } & \multicolumn{2}{l|}{ Itraconazole } & \multicolumn{2}{l|}{ Amphotericin B } & \multicolumn{2}{l|}{ Nystatin } \\
\cline { 2 - 12 } & No. & $\%$ & No. & $\%$ & No. & $\%$ & No. & $\%$ & No. & $\%$ \\
\hline C. albicans & 8 & 53.33 & 10 & 66.67 & 1 & 6.67 & 0 & 0.00 & 1 & 6.67 \\
\hline C. tropicalis & 21 & 77.78 & 22 & 81.48 & 3 & 12.00 & 3 & 11.11 & 4 & 14.81 \\
\hline C. parapsilosis & 4 & 57.14 & 4 & 57.14 & 0 & 0.00 & 1 & 14.29 & 1 & 14.29 \\
\hline C. glabrata & 6 & 85.71 & 6 & 85.71 & 1 & 14.29 & 1 & 14.29 & 2 & 28.57 \\
\hline C. krusei & 4 & 100.00 & 4 & 100.00 & 1 & 25.00 & 1 & 25.00 & 2 & 50.00 \\
\hline
\end{tabular}

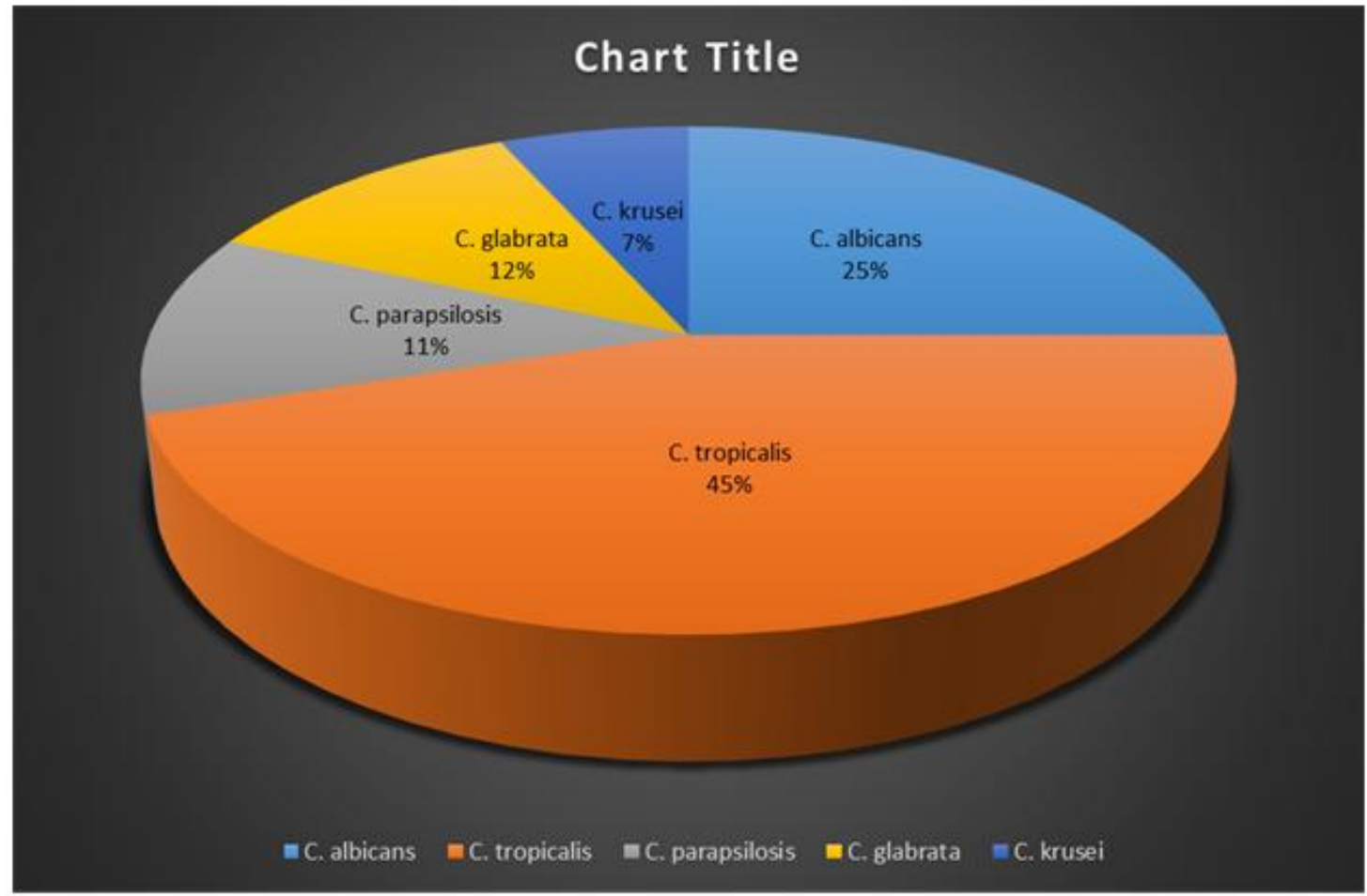




\begin{tabular}{|c|c|c|c|c|c|c|}
\hline S. no. & Patient Name & Age & Sex & ward & sample type & Risk factor \\
\hline 263 & kaka of harjinder & 1 & M & $\mathrm{NICU}$ & blood culture & SGA/Prematurity \\
\hline 12 & Kaka s/o kashmir & 1 & M & $\mathrm{NICU}$ & blood culture & very low birth wt. \\
\hline 27 & kaka s/o sikandar & 1 & M & $\mathrm{NICU}$ & blood culture & low birth wt. \\
\hline 90 & jashan & 1 & M & $\mathrm{NICU}$ & blood culture & prematurity \\
\hline 199 & gudiya of sita & 1 & $\mathrm{~F}$ & $\mathrm{NICU}$ & blood culture & low birth wt. \\
\hline 230 & kaka of ranjit & 1 & $M$ & $\mathrm{NICU}$ & blood culture & SGA/Prematurity \\
\hline 239 & kaka of dilbagh singh & 1 & $M$ & $\mathrm{NICU}$ & blood culture & SGA/Prematurity \\
\hline 240 & kaka of vijay & 1 & $M$ & $\mathrm{NICU}$ & blood culture & SGA/Prematurity \\
\hline 242 & gudiya of vikas & 1 & $\mathrm{~F}$ & $\mathrm{NICU}$ & blood culture & SGA/Prematurity \\
\hline 243 & kaka of bunty & 1 & M & $\mathrm{NICU}$ & blood culture & SGA/Prematurity \\
\hline 253 & gudiya of ranjit & 1 & $\mathrm{~F}$ & NICU & blood culture & SGA/Prematurity \\
\hline 257 & kaka of gurprit & 1 & M & NICU & blood culture & very low birth wt. \\
\hline 258 & shubham & 1 & M & NICU & blood culture & very low birth wt. \\
\hline 259 & kaka of jewan & 1 & M & $\mathrm{NICU}$ & blood culture & very low birth wt. \\
\hline 264 & kaka of vijay & 1 & M & NICU & blood culture & SGA/Prematurity \\
\hline 266 & gudiya of kashmir & 1 & $\mathrm{~F}$ & NICU & blood culture & low birth wt. \\
\hline 272 & gudiya of sujita & 1 & M & NICU & blood culture & low birth wt. \\
\hline 282 & kaka of akash & 1 & $M$ & $\mathrm{NICU}$ & blood culture & SGA/Prematurity \\
\hline
\end{tabular}

A total of $40 \quad(66 \%)$ out of 60 Candida species isolates obtained from the clinical isolates produced biofilm. Tissue culture plate method $(66.67 \%)$ was found to be more sensitive than Tube method (40\%) (p <0.05). Only 26.67\% ( 4 of 15) of $C$. albicans isolates produced biofilm, which was significantly $(\mathrm{p}<0.05)$ lower than the percentage of all non albicans Candida species isolates producing slime (88.89\%, 40 of 45$)$ which was according to the findings obtained in studies done by Shin et al., 2002 and Gokce et al., 2007. Strong biofilm production was seen in $C$. kruse $i$ and $C$. tropicalis. Weak biofilm production was seen in C. albicans. Biofilm producers were more resistant to antifungal drugs.

In conclusion, there is significant shift from C. albicans to Non albicans Candida species. Non albicans Candida species are

more commonly associated with antifungal resistance and biofilm production. So, it has become imperative for us to take timely steps for early speciation and antifungal susceptibility pattern of the clinically significant Candida isolates prevalent in our society. Early empirical antifungal therapies can also reduce the ICUs/NICUs associated mortality.

\section{References}

Agarwal, J., Bansal, S., Mailk, G.K., Jain, A. 2004. Trends in neonatal septicemia: Emergence of Nonalbicans Candida. Indian Pediatr., 41: 712-5.

Alka Nerurkar, Priti Solanky, Nilesh Chavda, Hinal Baria, Binita Desai. 2012. Isolation of Candida Species in clinical specimens and its virulence 
factor: The biofilm. Int. J. Med. Sci. Public Health, 1(2): 97-100.

Babin, D., Kotigadde, S., Rao, P.S., Rao, T.V. 2013. Clinico-mycological profile of vaginal candidiasis in a tertiary care hospital in Kerala. Int. $J$. Res. Biol. Sci., 3(1): 55-9.

Baradkar, V.P., Mathur, M., Kumar, S., Rathi, M. 2008. Candida glabrata emerging pathogen in neonatal sepsis. Ann. Trop. Med. Pub. Health, 1: 5-8.

Beck-Sague, C.M., Azimi, P., Fonseca, S.N., Baltimore, R.S., Powell, D.A., Bland, L.A., Arduino, M.J., McAllister, S.K., Huberman, R.S., Sinkowitz, R.L., et al. 1994. Blood stream infections in neonatal intensive care unit patients result of a multicentre study. Pediatr Infect. Dis. J., 13: 1110-1116.

Bonassoli, L.A., Bertoli, M., Svidzinski, T.I. 2005. High frequency of Candida parapsilosis on the hands of healthy hosts. J. Hosp. Infect., 59: 159-62. [PubMed: 15620452]

Chander, J. 2009. Candidiasis. In A text book of Medical Mycology. New Delhi: Mehta Publishers, 266-90.

Gokse, G., Cerkcioglu, N., Yagci, A. 2007. Acid proteinase, phospholipase and biofilm production of candida species isolated from blood culures. Mycopathologia, 164: 265-269.

Johnsson, H., Ewald, U. 2004. The rate of candidaemia in preterm infants born at a gestational age of 23-28 weeks is inversely correlated to gestational age. Acta Paediatr., 93: 954-958.

Juyal, D., Sharma, M., Pal, S., Rathaur, V. K., \& Sharma, N. 2013. Emergence of Non-Albicans Candida Species in Neonatal Candidemia. North American J. Med. Sci., 5(9), 541-545. http://doi.org/10.4103/19472714.118919
Kikani, K., Joshi, P., Mehta, S., Kilkani, B., Aring, B., Kamothi, M. 2010. Species distribution and antifungal susceptibility pattern in the cases of vaginal candidiasis in Saurashtra region of Gujarat. Electron $J$. Pharmac. Ther., 3: 9-1.

Kossoff, E.H., Buescher, E.S., Karlowicz, M.G. 1998. Candidemia in a neonatal intensive care unit: trends during fifteen years and clinical features of 111 cases. Pediatric Infect. Dis. J., 17: 504-508.

Narain, S., Shastri, J.S., Mathur, M., Mehta, P.R. 2003. Neonatal systemic candidiasis in a tertiary care Centre. IJMM, 21(1): 56-8.

Narang, A., Agarwal, P.R., Chakrabarti, A., Kumar, P. 1998. Epidemiology of systemic candidiasis in a tertiary care neonatal unit. J. Trop. Pediatrics, 44(2): 104-8.

Ng, K.P., Saw, T.L., Na, S.L., Soo-Hoo, T.S. 2001. Systemic Candida infection in University Hospital 1997-1999: the distribution of Candida biotypes and antifungal susceptibility patterns. Mycopathologia, 149(3): 141-6.

Park, K. 2011. Health information and basic medical statistics. In: Park's textbook of preventive and social medicine. 21st ed. India: Bansari Das Bhanot, p. 779-92.

Sardana, V., Pandey, A., Madan, M., Goel, S.P., Asthana, A.K. 2012. Neonatal candidemia: A changing trend. Indian J. Pathol. Microbiol., 55: 132-3. [PubMed: 22499329]

Shin, J., Kee, S., et al. 2002. Biofilm production by isolates of Candida species recovered from nonneutropenic patients: comparison of bloodstream isolates from other sources. J. Cl. Microbiol., 40(4): 1244-48. 
Sobel, J.D. 2007. Vulvovaginal candidosis. Lancet Lond Engl., 9; 369(9577): 1961-71.

Trofa, D., Gacser, A., Nosanchuk, J.D. 2008. Candida parapsilosis; an emerging fungal pathogen. Clin. Microbiol. Rev., 21(4): 606-25.

Wayne, A. 2009. Method for Antifungal Disk Diffusion Susceptibility Testing of Yeasts. CLSI M44-A2 ISBN 156238-703-0).

Xess, I., Jain, N., Hasan, F., Mandal, P., Banerjee, U. 2007. Epidemiology of candidemia in a tertiary care Centre of North India: 5-year study. Infect., 35: 256-9. [PubMed: 17646917]

\section{How to cite this article:}

Rajesh Bansal, Loveena Oberoi, Kanwardeep Singh and Pushpa Devi. 2016. Species Distribution, Biofilm Formation and Antifungal Susceptibility of Candida Isolates in Blood Samples of NICU Patients at Tertiary Care Centre, Amritsar, India. Int.J.Curr.Microbiol.App.Sci. 5(12): 628-634. doi: http://dx.doi.org/10.20546/ijcmas.2016.512.069 